\title{
C.T. Aided Stereotaxy for Depth Electrode Implantation and Biopsy
}

\author{
T.M. Peters and André Olivier
}

SUMMARY: We describe a computer program which facilitates the analysis of a series of C.T. scans made while a stereotaxic frame is fixed to the patient.

The program has 2 modes of operation:

a) The operator may select a region and determine the three-dimensional frame coordinate.

b) The operator may select a set of frame coordinates and have the computer program display these at the appropriate sites on the C.T. scans. If these sites are the positions of depth electrodes, then a recording of the epileptic spike activity may be displayed at the appropriate sites on the scans.

RÉSUMÉ: Les auteurs décrivent un programme d'ordinateur pour analyser une série de tomographies axiales faites en conditions stéréotaxiques.

Le programme fonctionne selon deux modes:

a) L'opérateur peut sélectionner une cible intracérébrale et en obtenir instantanément les coordonnées tridimensionnelles du cadre.

b) L'opérateur peut sélectionner les coordonnées du cadre de sorte que l'ordinateur indique sur les coupes tomographiques le site exact des cibles choisies. Si ces sites correspondent à la position d'électrodes profondes, l'enregistrement de l'activité épileptique peut dès lors être représenté de façon graphique aux sites anatomiques sur les coupes tomographiques.

Can. J. Neurol. Sci. 1983; 10:166-169

We describe a computer program that facilitates analysis of a series of C.T. scans made with a stereotaxic frame fixed to the patient. The system allows C.T. features to be readily identified by the user with the program automatically calculating the appropriate frame co-ordinates. Alternatively, the user may specify a set of frame co-ordinates and the computer will identify these points on the appropriate C.T. images. If the positions of implanted depth electrodes are to be determined, then the electrodes and recording sites may be accurately plotted on the images. If desired, the level of epileptiform activity and/or the number of seizures recorded during the period of implantation may be displayed on the image and related to specific pairs of recording sites.

\section{MATERIALS AND METHODS}

At the Montreal Neurological Institute, stereotaxic surgical techniques are used for many procedures, including biopsy of tumours, aspiration of cystic cavities, possible insertion of radioactive sources, and implantation of depth electrodes for recording the spatial distribution of seizure activity (Ives et al 1974, Olivier et al 1982c). For these procedures a modified LEKSELL stereotaxic frame (Tipal Instruments, Inc., Montreal) is used (Bertrand et al 1965). On this frame various specialized surgical tools may be mounted (Olivier et al $1982 \mathrm{a}$, b). In addition computer programs have been devised to assist the neurosurgeons in stereotaxic procedures (Bertrand et al 1974, Thompson et al 1972).

Graduated $\mathrm{cm}$ markers on the stereotaxic frame allow the positions of structures within the brain to be specified by $(x, y, z)$ co-ordinates. Use of a tele-radiography system (Olivier et al
$1982 \mathrm{~b}$ ) installed in the operating theatre provides $\mathrm{x}$-ray images of the brain and the frame with a minimum of differential magnification between the proximal and distal regions of the brain. The co-ordinate system engraved on the frame's support structure is lead-filled and can be easily read on the radiograph. Measurements of 3-dimensional co-ordinates of specific structures may thus be determined to within a millimetre by reading directly from A-P and lateral films.

While the system described above allows for accurate localization of structures that can be uniquely visualized with two orthogonal views, there are many instances in which tumours or cysts cannot be adequately located using the standard techniques of arterial and venous phase angiography and ventriculography. C.T. scanning however, generally demonstrates such lesions with ease. In such cases it is therefore important that a series of C.T. scans be made of the patient with the frame rigidly fixed to the skull. It must be possible to analyse these images accurately so that the frame co-ordinates of individual structures may be obtained. This paper describes our solution. The system we have devised determines the frame co-ordinates of observed structures. It also allows the operator to specify sets of frame co-ordinates and have these automatically displayed or super-imposed upon the appropriate C.T. scan cross-sections. In cases where a record of the positions of implanted electrodes is needed, the position of each recording site can be displayed on the appropriate C.T. image. The multi-channel record of EEG activity from each of the recording sites can be analysed separately (Olivier et al 1982a, Gotman et al 1979), and the results expressed in terms of seizure activity at the appropriate sites. These results are plotted on the image as numbers of seizures occurring between selected pairs of recording sites. 
The program also helps determine the axial rotation, lateral tilt and mediolateral displacement of the frame in relation to the head.

\section{Modification of Stereotaxic Frame}

A number of reports in the literature (Boëthius et al 1980, Cacak et 1982, Brown 1979, Gleason et al 1978, Leksell et al 1980, Perry et al 1980, Lunsford et al 1982, Cohadon et al 1977) have described apparatuses adapted to interact with C.T. We have chosen to stay with the LEKSELL geometry as far as possible in order to maintain consistency with existing procedures. Both the standard LEKSELL frame and the modified "MNI" frame are made from $1 / 2$ " aluminum bars, and are rigidly fixed to the patient's skull by means of 3 steel pins which are held in place by steel chucks mounted on the frame. Dense metallic objects do not image well in C.T. scanners due to their high radio opacity at diagnostic $x$-ray beam energies (Joseph 1981). Typical artefacts include inaccurate or over-ranged C.T. number readings, "incomplete" or "inconsistent-data" artefacts causing streaking across the image, and beam hardening artefacts induced by spectral hardening of the $\mathrm{x}$-ray beam (Boëthius et al 1980, Perry et al 1980, Bertrand et al 1965). In order to use our frame in our C.T. scanner (EMI 5005), we replaced the steel chucks and pins with aluminum equivalents. Although these changes reduced the artefact level significantly, we are currently redesigning the frame to eliminate residual artefacts by replacing the existing aluminum side supports with plexiglass and the aluminum pins with carbon fibre rods.

With the standard frame, it is impossible togauge longitudinal placement or orientation within the C.T. scanner. We have therefore constructed a pair of removable plexiglass side plates with embedded aluminum rods set in a " $Z$ " pattern (Fig. 1). If the frame is positioned horizontally within the scanner, the images of the upper and lower rods in each plate serve as fiducial markers on the C.T. scan. This allows the correct scale and orientation of the frame within the C.T. scan field to be determined. The images of the oblique bars on either side are used to determine the level of the C.T. slice with respect to the frame co-ordinates. The plates containing the rods are secured

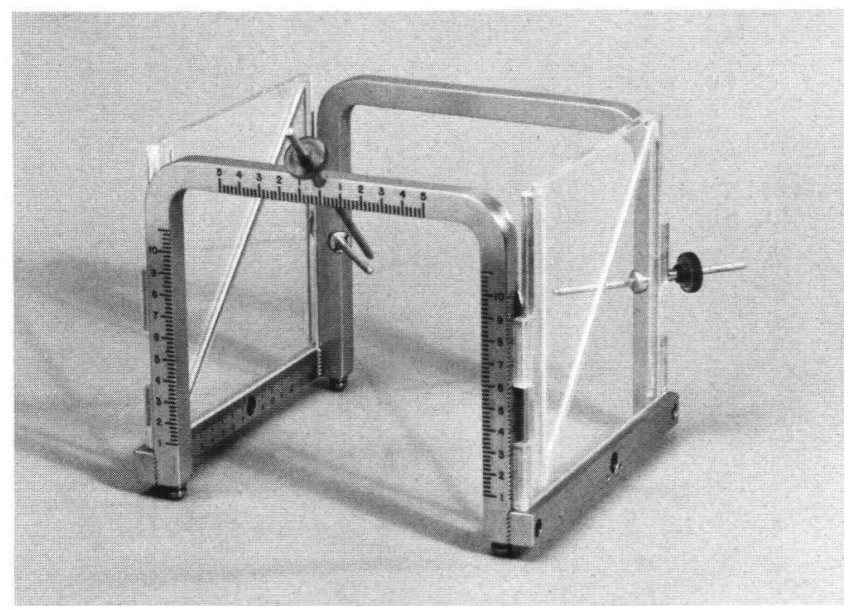

Figure 1 - Modified LEKSELL frame with removeable plexiglass side plates. Embedded aluminum rods are set in a " $Z$ " pattern. The oblique bars are used to determine the level of the C.T. slice with reference to the frame coordinates. to the frame by means of a precision-milled slot and rail system to ensure that rod position relative to the frame is precisely known. Since the program does not check for a tilt of the frame in the axial direction, it is extremely important to ensure that the frame is secured in a true horizontal position relative to the C.T. scanner axis.

\section{Operation of Analysis Program}

Although the original scans are produced by an EMI 5005 scanner, we find it convenient and more flexible to perform the subsequent analysis on a separate computer system. We have implemented such a program in FORTRAN-4 on a Digital Equipment Corp PDP-11/60 equipped with a Lexidata image display and utilizing the RSX-11M operating system.

The program is divided into a number of sections. The first is a simple transfer step to read the required scans from an EMI archive tape and place them on the disc storage of the PDP-11. Once the images have been placed in the disc files, the first in the series is requested by the operator and is displayed on the screen. Using a track-ball controlled cursor, the operator 'points' to the neighbourhood of the images of the four axial fiducial rods. The computer determines the exact centres of each rod and also the locations of the oblique rods on either side. From the positions of these latter rods the system identifies the axial level of the particular slice being viewed. After the operator has 'guided' the program as to the positions of the fiducial markers on the first image of the series, the program then automatically interrogates the remainder of the images and sets up files which identify the level and coordinate system appropriate to each slice.

This set of information is sufficient to allow frame coordinates to be directly related to image coordinates in subsequent phases of the program. When any image is displayed after this first step, it has superimpcsed upon it a set of $\mathrm{cm}$ markings corresponding to the engraved frame coordinates.

The program is menu driven and allows the operator the choice of one of the following two principle modes.

A. Determination of frame coordinates of identified structures.

This mode is used primarily to establish the frame coordinates of a target volume which will be the subject of a stereotaxically guided biopsy or aspiration. The frame coordinate(s) of the points which are identified at this step appear superimposed on the appropriate image(s) (Fig. 2) as well as the operators console.

B. Determination of image coordinates corresponding to specified frame coordinates.

This mode is useful when establishing the relationship of stereotaxically implanted electrodes to the transverse anatomy. This section has two sub-modes. One may type in a series of frame coordinate triplets and simply have each set displayed on its appropriate transverse image. Alternatively, one may specify that the coordinates entered are the distal tips of implanted electrodes and label them accordingly. Since implants at this Institute are performed as far as possible in transverse planes relative to the frame, the electrode trajectories along with the positions of the active recording sites may be plotted on the appropriate images (Fig. 3). If necessary, physiological data relating to epileptogenic activity may also be superimposed on the 


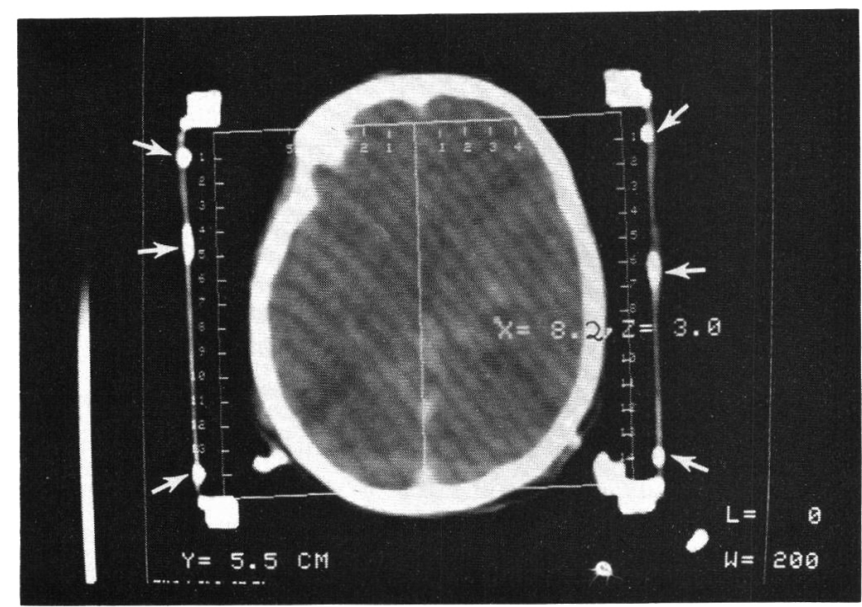

Figure 2 - C.T. image with computer generated frame coordinates. The center of the lesion is selected by positioning the diplay system cursor at the appropriate position. It is identified on the image by a white marker. The lesions' frame coordinates are superimposed on the image. The images of the ' $Z$ " bars, used as fiducial markers by the computer, are indicated by the arrows in the image.

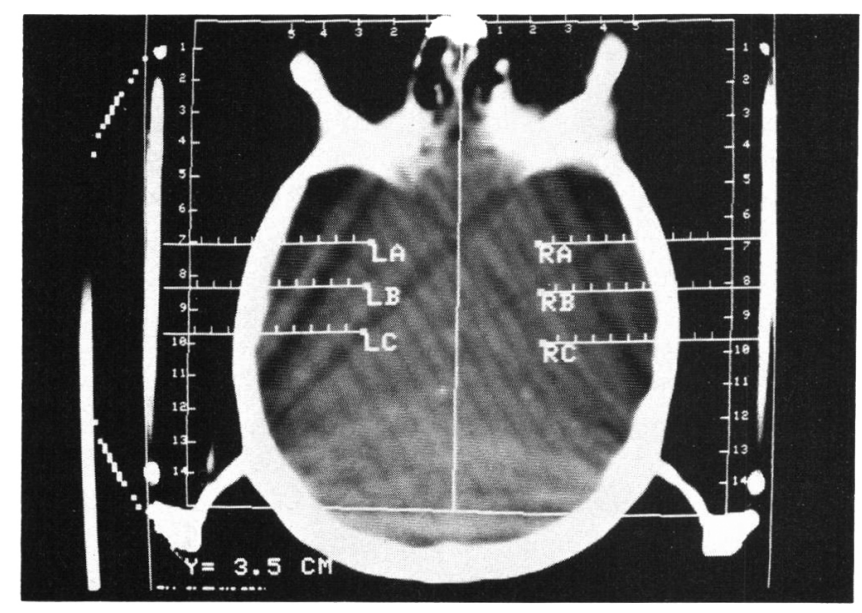

Figure 3-Locations of implanted electrodes and active recording sites displayed on the image.

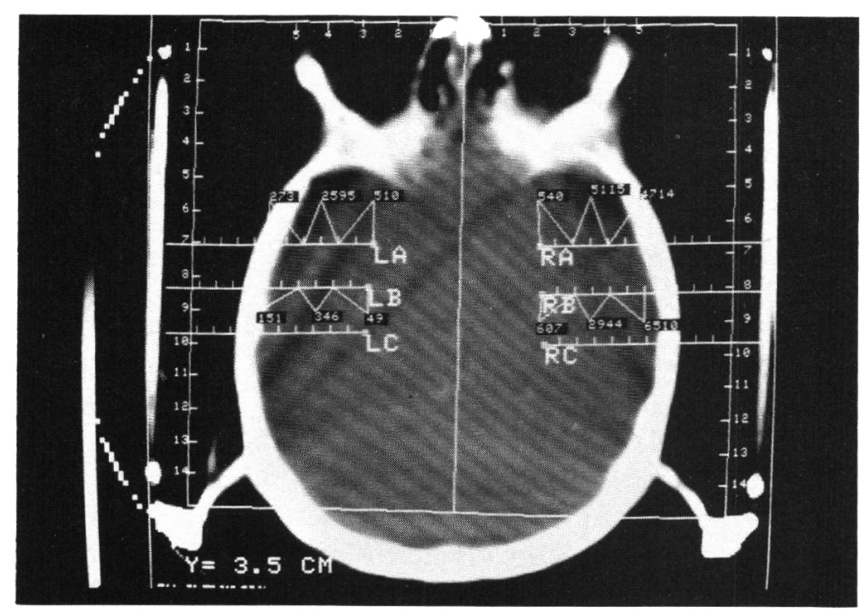

Figure 4- Graphic demonstration of epileptic spike activity between identified recording sites. Indicated here is the number of spikes recorded between selected electrode pairs over a 12 day period. image (Fig. 4). Apart from the display-system routines, the program is written in FORTRAN, and should be fairly transportable between different computer systems. Readers interested in adapting this sytem to their own requirements are invited to contact the authors.

\section{Accuracy of Frame Measurement}

In order to verify the accuracy with which C.T. visualizes the position of structures within the frame, we mounted a number of $2 \mathrm{~mm}$ glass beads within the frame on a series of wires. The frame was then radiographed by the standard teleradiographic system (FSD $4.8 \mathrm{~m}$ ) used at this Institute, in both A-P and lateral projections. Using the standard procedure of reading the co-ordinates directly from the films, the 3-dimensional coordinates of each of these beads were determined. The frame was subsequently scanned in the EMI 5005 scanner using technique factors identical to those used to scan a patient. In this instance the diameter of our scan field was $320 \mathrm{~mm}$, and the matrix was $160 \times 160$, giving pixel size of approximately $2 \mathrm{~mm}^{2}$. This pixel size was selected rather than a $1 \mathrm{~mm}^{2}$ resolution, in order to avoid over-range artefacts in the images of the fiducial marker rods. (This occured occasionally at the higher resolution.) In future the rod material will be changed to avoid this problem. The thickness of each slice was $8 \mathrm{~mm}$; the inter-slice distance was $4 \mathrm{~mm}$. Table 1 lists these results. The measurements of bead positions made with the teleradiographs, and those determined by computer analysis of the C.T. scans, all agreed within the limits of accuracy determined by the pixel size and slice thickness (i.e. $\pm 1 \mathrm{~mm}$ in the plane of the image and \pm $4 \mathrm{~mm}$ in the axial direction). Higher resolution reconstruction and narrower slices will reduce these uncertainities to $\pm .5 \mathrm{~mm}$ and $\pm 2 \mathrm{~mm}$ respectively.

\section{RESULTS}

We present here examples of two clinical cases in which this system was used. The first was a 21-year-old woman with a fluid-filled cyst in the right hemisphere. In order to determine the co-ordinates of the centre of the lesion, a series of C.T. scans was made and analysed in the manner described above (Fig. 2). The co-ordinates of the centre of the cyst were also determined by careful analysis of a series of teleradiographic angiograms made with the frame in place, showing agreement with the computer calculations, within the limits demonstrated by Table 1 .

The second patient was a 21-year old woman diagnosed as having bitemporal cerebral seizures. To determine the side of

Table 1: Comparison of X-ray and C.T. measured coordinates

\begin{tabular}{|c|c|c|c|c|c|c|}
\hline \multirow[b]{2}{*}{ Bead No. } & \multicolumn{2}{|c|}{$\mathbf{X}$ coordinate } & \multicolumn{2}{|c|}{ Y coordinate } & \multicolumn{2}{|c|}{$\mathbf{Z}$ coordinate } \\
\hline & $X$-ray & C.T. & $X$-ray & C.T. & $X$-ray & C.T. \\
\hline 1 & $3.3 \mathrm{~cm}$ & $3.2 \mathrm{~cm}$ & $9.4 \mathrm{~cm}$ & $9.4 \mathrm{~cm}$ & $5.3 \mathrm{~cm}$ & $5.3 \mathrm{~cm}$ \\
\hline 2 & $6.2 \mathrm{~cm}$ & $6.1 \mathrm{~cm}$ & $8.3 \mathrm{~cm}$ & $8.1 \mathrm{~cm}$ & $2.3 \mathrm{~cm}$ & $2.4 \mathrm{~cm}$ \\
\hline 3 & $1.6 \mathrm{~cm}$ & $1.7 \mathrm{~cm}$ & $8.0 \mathrm{~cm}$ & $8.1 \mathrm{~cm}$ & $4.2 \mathrm{~cm}$ & $4.2 \mathrm{~cm}$ \\
\hline 4 & $3.5 \mathrm{~cm}$ & $3.5 \mathrm{~cm}$ & $6.8 \mathrm{~cm}$ & $6.6 \mathrm{~cm}$ & $2.0 \mathrm{~cm}$ & $2.2 \mathrm{~cm}$ \\
\hline
\end{tabular}

Note that the pixel width is $2 \mathrm{~mm}$ and the slice width is $8 \mathrm{~mm}$ giving an expected accuracy of $\pm 1 \mathrm{~mm}$ in $X$ and $Z$, and $\pm 4 \mathrm{~mm}$ in the $Y$ measurements. There is an estimated error of $\pm 1 \mathrm{~mm}$ in the $x$-ray film readings. 
seizure onset and to specify the extent of seizure activity 10 depth electrodes, each with 10 recording sites, were implanted bilaterally, 3 in each temporal lobe and 2 in each frontal lobe. Sixteen channels of EEG were recorded from selected sites over a period of 12 days (Ives et al 1974). Prior to the insertion of the electrodes, the patient's head was scanned while in the frame, and a series of 5 C.T. slices were recorded. These covered the region of the proposed electrode sites. The position of the distal ends of each electrode were subsequently entered into the computer. The labeling scheme used was RA, RB, RC for the right temporal positions and LA, LB and LC for the left temporal positions. Similar labeling conventions were used for the frontal lobes. In addition to accurately displaying the position of each of the 100 potential recording sites (Fig. 3), the system can also be used to specify the level of epileptogenic activity in various regions by indicating the frequency of interictal spikes and/or the location and number of seizure onsets (Ives et al 1974, Gotman et al 1979). In the example given here the number of seizures occurring at the appropriate pairs of electrode recording sites is displayed in the image. Thus, an accurate map of seizure activity related to the patient's anatomy is permanently recorded (Fig. 4).

\section{Discussion}

We have demonstrated the advantages of combining stereotaxic surgical techniques with the C.T. scanner and related computer systems. We have barely scratched the surface of this technique and envisage its future extension in the following areas:

1. Identification of the target volume and computer assisted determination of the optimum approach for biopsies of tumours and aspiration of cysts, or for radio-isotope implantation.

2. Generation of vertical views (sagittal or coronal) from the series of transverse slices and correlation of these views with digital angiograms.

3. Accurate determination of the $\mathrm{Ca}-\mathrm{Cp}$ (anterior-posterior commissure) line so that standard brain maps (traditionally defined parallel and perpendicular to this line) may be correlated with and superimposed upon C.T. scans and angiograms.

4. Refinement of the stereotaxic procedure, so that the positions of probes and cannulae relative to the patient's anatomy can be viewed by the surgeon during the operation.

5. Development of stereotaxic procedures in Nuclear Magnetic Resonance Imaging.

\section{ACKNOWLEDGMENT}

We would like to thank Charlotte Payette for her assistance in the preparation of the manuscript.

\section{REFERENCES}

Bertrand, G. and Jasper, H. (1965) Microelectrodes recording of unit activity in the human thalamus. Conf Neurol, 26:205-208.

Bertrand, G. and Olivier A. (1974) Computer display of stereotaxic brain maps and probe tracts. Acta Neurochirur, supp. 21:235-243, (Springer-Verlag)

Boethius, J., Bergstrom, M. and Greitz, T. (1980) Stereotaxic computerized tomography with a GE 8800 Scanner. J Neurosurg 52:794-800.

Brown, R.A. (1979) A computerized tomography - computer graphics approach to stereotaxic localization. J Neurosurg 50:715-720.

Cacak, R.K. and Law, J.D. (1982) A stereotaxic adapter compatible with computer tomography. Radiology 143:271.

Cohadon, F., Rougier, A., DA Silva Nunes Neto, D., Pigneux, J., Caille, J.M. and Constant, P. (1977) La tomodensitométrie en conditions stéréotaxiques. Neurochir 23 (1977), 433-452.

Gleason, C.A., Wise, B.L. and Feinstein, B. (1978) Stereotactic localization (with computed tomographic scanning), biopsy and radiofrequency treatment of deep brain lesions. Neurosurgery 2:272-222

Gotman, J., Ives, J.R. and Gloor, P. (1979) Automatic recognition of interictal epileptic activity in prolonged EEG recordings. Electroenceph clin Neurophysiol 546:510-520.

Ives, J.R., Thompson, C.J., Gloor, P., Olivier, A. and Woods, J.F. (1974) The on-line computer detection and recording of spontaneous temporal lobe epileptic seizures from patients with implanted depth electrodes via a radiotelemetry link. EEG J 37:205. (Abst.)

Joseph, P.M. (1981) Artifacts in computed tomography. Chapt 114 in "Radiology of the skull and brain" Vol. 5, Technical aspects of computed tomography. T.H. Newton \& D.C. Potts eds. C.V. Mosby, N.Y. pp. 3956-3991.

Leksell, L. and Jernberg B. (1980) Stereotaxis and tomography, a technical note. Acta Neurochir (WIEN) 52:1-7.

Lunsford, L.D., Rosenbaum, A.E. and Perry J. (1982) Stereotactic surgery using the "therapeutic" C.T. Scanner. Surg Neurol 18:116-122.

Olivier, A. and Bertrand, G. (1982)(a) Stereotaxic device for percutaneous twist-drill insertion of depth electrodes and for brain biopsy. J. Neurosurg 56:307-308.

Olivier, A. and Bertrand, G. (1982)(b) Stereotaxic implantation of depth electrodes for seizure recording. MNI Publications, Montreal, Quebec.

Olivier, A., Gloor, P., Andermann, F. and Ives, J.R. (1982)(c) Occipitemporal epilepsy studied with stereotaxically implanted depth electrodes and successfully treated by temporal resection. Ann Neurol 11:428-432.

Perry, J.H., Rosenbaum, Lunsford L.D., Swink, C. A. and Zorub, D.S. (1980) Computed Tomography - guided stereotactic surgery: conception and development of a new stereotaxic methodology. Neurosurgery, 7:376-381.

Thompson, C.J. and Bertrand, G. (1972) A computer program to aid the Neurosurgeon to locate probes used during stereotaxic surgery on deep cerebral structures. Comput Programs Bio Med 2:265-276. 